\title{
Effects of Nanoscale Clustering in Amorphous Carbon
}

\author{
J. D. Carey and S. R. P. Silva
}

Nanoelectronics Centre, Advanced Technology Institute, School of Electronics and Physical Sciences, University of Surrey, Guildford, GU2 7XH, U.K.

David.Carey@surrey.ac.uk

Summary. In this review the effects of clustering associated with the $\mathrm{sp}^{2}$ and $\mathrm{sp}^{3}$ phases of amorphous carbon thin films are examined. We highlight that many of the optical and electronic properties of these films can be explained by consideration of disorder in the $\mathrm{sp}^{2}$ phase. Within the context of topological and structural disorder, we explain the variation of the visible Raman line width, Raman shift, Tauc gap and Urbach energy as a function of deposition conditions. We further go on to describe how intra-sp ${ }^{2}$ cluster interactions are responsible for the narrowing of the electron paramagnetic resonance line width with increasing spin density and how this intracluster interaction can be extended to the intercluster transport properties, in particular, for electron field emission from the films. We also examine how the mechanical properties of carbon films are affected by clustering which can be enhanced by thermal annealing.

\section{Introduction and Bonding in Carbon}

Crystalline carbon is unique amongst the elements of the periodic table being able to form one of the hardest naturally occurring materials - diamond, and also one of the softest - graphite. From an electronic point of view these two materials possess, respectively, a $5.5 \mathrm{eV}$ energy band gap and a zero band gap, making the electronic properties vary between those found in an insulator and those of a semi-metal. This very different behaviour can be traced to the bonding and the bond hybridization that are present - as shown in Fig. 1. In the case of $\mathrm{sp}^{3}$ hybridization, four $\sigma$ bonds, arranged at $109.5^{\circ}$ to each other allow for the tetrahedral bonding of diamond. It is these four bonds that are responsible for the high hardness and large energy band gap. By contrast, in the case of $\mathrm{sp}^{2}$ hybridized carbon there are three $\sigma$ bonds bonding in-plane to three other carbon atoms at $120^{\circ}$ to each other. In addition, there is one $\mathrm{p}_{\mathrm{z}}$ orbital perpendicular to the plane of the threefold bonding. This anisotropy in the bonding distinguishes the in-plane electronic properties of graphite from the properties perpendicular to the basal plane. Whilst there is a strong inplane interaction, the weaker out-of-plane interactions are responsible for the lubricant properties of graphite.

These bond hybridizations are not restricted to the crystalline forms of carbon and are available to form amorphous carbon films (Fig. 1) which have a wide range of properties, some of which are presented in table 1. 


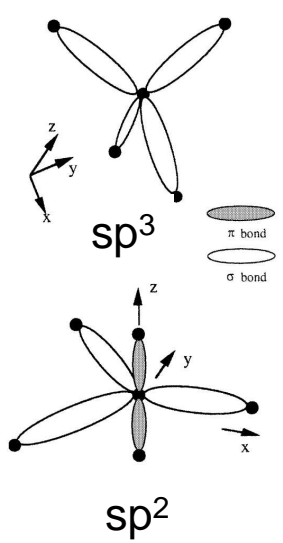

Bonding
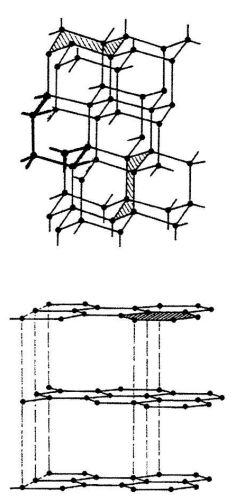

Crystalline

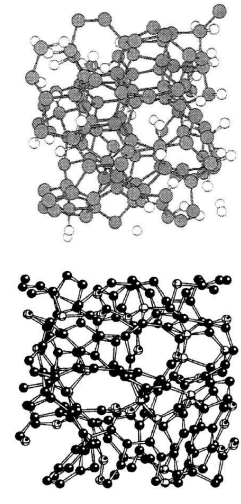

Amorphous

Fig. 1. A schematic representation of different types of hybridized bonding and the resultant crystalline and model amorphous structures.

It is important at the outset to distinguish between the different types of amorphous carbon. For example, from table 1 the physical and electronic properties of tetrahedral amorphous carbon (ta-C) are very different from hydrogenated diamond-like carbon (DLC:H) even though they both a similar $\mathrm{sp}^{3}$ fraction. In the case of the former, the $\mathrm{sp}^{3}$ content is mainly made up of $\mathrm{C}-\mathrm{C}$ bonds which result in a high hardness thin film whereas in the latter the $\mathrm{sp}^{3}$ content also contains $\mathrm{C}-\mathrm{H}$ bonding in addition to $\mathrm{C}-\mathrm{C}$ bonding. In this review the terms amorphous carbon, a-C, and hydrogenated amorphous carbon, a-C:H, will be used as generic terms for disordered carbon either $\mathrm{H}$ free or $\mathrm{H}$ containing. For specific types of films, terms such as diamond-like carbon or polymer-like carbon will be used. [1]

\begin{tabular}{|c|c|c|c|c|c|}
\hline Category & $\mathrm{sp}^{3}(\%)$ & $\begin{array}{c}\text { Optical bandgap } \\
(\mathrm{eV})\end{array}$ & $\mathrm{H}($ at.\% $)$ & $\begin{array}{c}\text { Density } \\
\left(\mathrm{g} \mathrm{cm}^{-3}\right)\end{array}$ & $\begin{array}{c}\text { Hardness } \\
(\mathrm{GPa})\end{array}$ \\
\hline polymer-like a-C PAC & $60-80$ & $2.0-5.0$ & $40-65$ & $0.6-1.2$ & soft \\
graphitic-like a-C GAC & $0-30$ & $0.0-0.6$ & $0-40$ & $1.2-2.0$ & soft \\
diamond-like a-C DLC & $40-60$ & $0.8-4.0$ & $20-40$ & $1.5-3.0$ & $20-40$ \\
tetrahedral a-C ta-C & $65-90$ & $1.6-2.6$ & $0-30$ & $2.5-3.5$ & $40-65$ \\
\hline
\end{tabular}

Table 1. Physical properties of different forms of a-C thin films.

One of the main driving forces for the technological use of amorphous carbon thin films is the ability to grow thin films over large area, at low temperatures with excellent uniformity. The low temperature deposition, of- 
ten performed using plasma enhanced chemical vapour deposition (PECVD) allows the growth onto non-conventional substrates such as organics or glass substrates. The excellent uniformity of the physical and electronic properties is also matched by the mirror-smooth quality of the films. Extensive atomic force microscopic studies of a-C and a-C:H films have shown that they typically have a rms roughness of less than $1 \mathrm{~nm}$. This makes a-C films ideal for microelectromechanical systems and for hard disk coatings. In addition, $\mathrm{a}-\mathrm{C}$ is believed to exhibit excellent biocompatibility and a-C coated surgical implants have been demonstrated. Less success has been forthcoming in the development of active electronic applications of a-C and a-C:H films [2] and as a consequence it is necessary to examine the electronic properties and the effects of disorder and localization at a nanometer level. To that end, this article first deals with the physics associated with the disorder associated with the $\mathrm{sp}^{2}$ phase and then extends the discussion to the intercluster transport properties of the films. Finally, the field emission from the carbon system is discussed.

\section{Disorder in Amorphous Carbon}

Amorphous carbon can be considered as a disordered mixed phase material consisting of a conductive $\mathrm{sp}^{2}$ phase, embedded in a less conductive $\mathrm{sp}^{3}$ matrix. The $\mathrm{sp}^{2}$ hybridised carbon atoms are usually in the form of nanometersized clusters mainly in the form of rings or olephinic chains. These $\mathrm{sp}^{2}$ clusters give rise to occupied $\pi$ and unoccupied $\pi^{*}$ bands with the separation between the bands being related to the size of the cluster. The $\mathrm{sp}^{3}$ component tends to be in the form of aliphatic chains of $\mathrm{C}$ atoms with $\mathrm{C}-\mathrm{C}$ and $\mathrm{C}-\mathrm{H}$ bonding being present. The optoelectronic properties of a-C and a-C:H films can be described in terms of transitions between the occupied $\pi$ states to the unoccupied $\pi^{*}$ states. Previous studies have shown [3] that it is possible to represent the occupied $\pi$ and unoccupied $\pi^{*}$ bands by Gaussian functions centred at $\mathrm{E}_{\pi\left(\pi^{*}\right)}$. The $\sigma$ and $\sigma^{*}$ states, which are associated with $\mathrm{sp}^{3} \mathrm{C}$ bonding lie further separated from each other. By deposition on suitable transparent substrates, such as Corning glass, it is possible to extract the parabolic Tauc gap and the exponential Urbach energy.

- The Tauc band gap corresponds to optical transitions between extended states and for the a-C system in the absence of disorder, the Tauc gap is given by the separation between the $\pi-\pi^{*}$ bands with a magnitude of $2 \mathrm{E}_{\pi}$. The larger the average size of the $\mathrm{sp}^{2}$ cluster, the smaller the Tauc gap.

- An alternative band gap that is sometimes employed is to measure the energy at which the absorption coefficient is $10^{4} \mathrm{~cm}^{-1}$; this is known as the $\mathrm{E}_{04}$ gap. Values associated with the $\mathrm{E}_{04}$ gap tend to be larger than the Tauc gap, as the absorption at $10^{4} \mathrm{~cm}^{-1}$ tends to occur in states beyond 
the $\pi$ and $\pi^{*}$ band edges. $\mathrm{E}_{03}$ and $\mathrm{E}_{05}$ gaps are also sometimes quoted in the literature.

- Transitions between extended-to-localised states in amorphous semiconductors are usually characterised by the Urbach energy, $E_{\mathrm{u}}$. The value of $\mathrm{E}_{\mathrm{u}}$ is found by fitting an exponential function to the slope of the absorption edge and has been used as a measure of disorder in other material systems. For example, in the case of low defect density a-Si:H the valence band tail has an exponential slope of about $45 \mathrm{meV}$, whereas the slope of the conduction band is $25 \mathrm{meV}$. In this manner the observed Urbach energy of $55 \mathrm{meV}$ for a-Si:H is mainly associated with the valence band and with low lying defect states.

Fanchini and colleagues have, however, suggested that while the experimentally observed values can be extracted, the validity of this approach for this dual mixed phase system may be too simplistic. As a result care must be exercised in the interpretation of the Tauc band gap and the Urbach energy that are obtained. [4] A second measure of disorder, based upon bond angle distortion, is the full width half maximum of the Raman active $\mathrm{G}$ band, $\Gamma_{\mathrm{G}}$. This Raman signal is found at around $1580 \mathrm{~cm}^{-1}$ and originates from the $\mathrm{E}_{2 \mathrm{~g}}$ vibration between $\mathrm{sp}^{2} \mathrm{C}$ bonds [5]. Several complementary optical [6-8] and Raman studies $[7,9]$ have revealed that as the Tauc gap increases the Urbach energy increases monotonically, but, that the width of the $\mathrm{G}$ band undergoes a maximum of about $1.5 \mathrm{eV}$, as shown in Fig. 2.
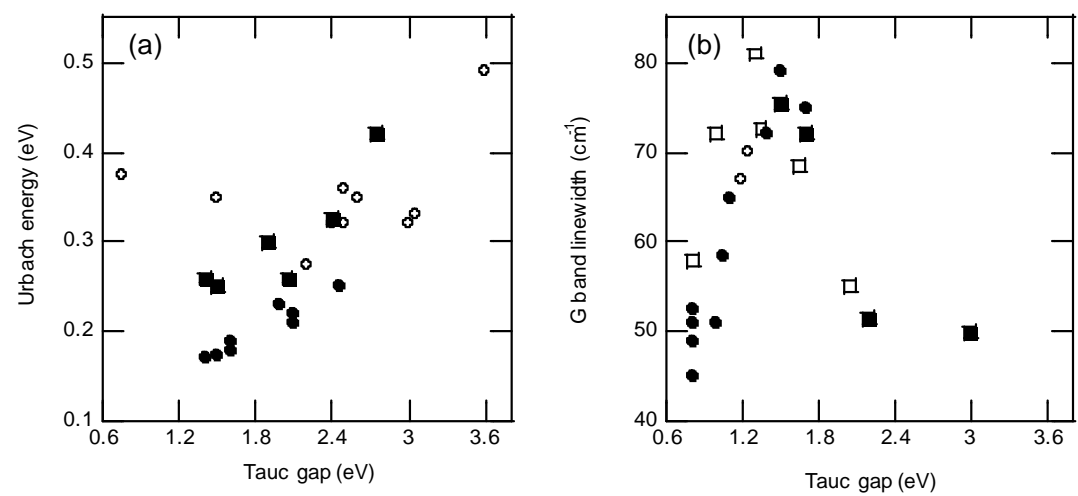

Fig. 2. Variation of (a) Urbach energy and (b) linewidth of Raman G band for different a-C films as a function of optical Tauc gap. The different symbols refer to different sets of films, see ref. [2].

In this respect if the Urbach energy and the width of $\mathrm{G}$ band are valid measures of disorder in the a-C:H system, then they must measure different aspects or types of disorder. Robertson previously proposed that the Urbach 
energy is a measure of an inhomogeneous disorder associated with different $\mathrm{sp}^{2}$ cluster sizes, whereas, the $\mathrm{G}$ band width has been attributed to a homogeneous disorder associated with bond angle disorder [10]. However, such an interpretation is unable to explain

(i) why the largest values of $\mathrm{E}_{\mathrm{u}}$ are observed at the highest Tauc gaps (Fig. 2(a)) since the cluster sizes are smallest there is only a small limited number of different cluster conformations possible,

(ii) the observation of a maximum in the $\mathrm{G}$ band line width with Tauc gap (Fig. 2(b)),

(iii) why the $\mathrm{G}$ band position is also dependent on the excitation wavelength, with the $\mathrm{G}$ band position tending to saturate at $\sim 1600 \mathrm{~cm}^{-1}$ for excitation wavelengths below $350 \mathrm{~nm}$ and the peak shifting to lower energies at longer wavelengths, as shown in Fig. 3(a), and finally

(iv) the observation that the $\mathrm{G}$ band line width depends on the excitation wavelength with the narrower values of $\Gamma_{\mathrm{G}}$ observed at increasing excitation energy as seen in Fig. 3(b).
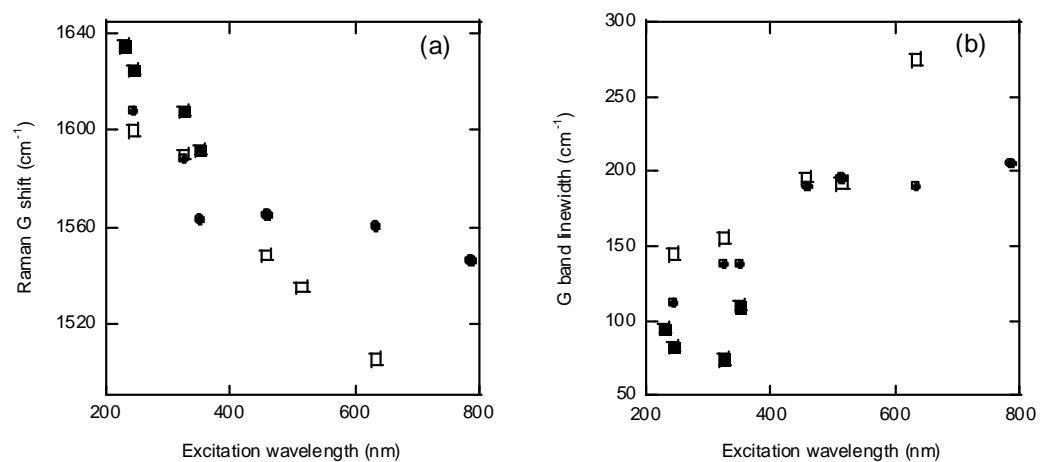

Fig. 3. Variation of (a) G band Raman shift and (b) G band linewidth for a series of polymer-like a-C:H films $(\boldsymbol{\square})$, diamond-like a-C:H $(\square)$ and graphitic carbon $(\bullet)$ thin films as a function of Raman excitation wavelength. (see ref. [5]).

Since the Raman spectra are a result of resonant excitation of $\mathrm{sp}^{2}$ clusters, dispersion of the $\mathrm{G}$ peak reflects excitation of clusters with different band gaps. The saturation of the $\mathrm{G}$ band position at $\sim 1600 \mathrm{~cm}^{-1}$ in Fig. 3(a) reflects the maximum possible Raman shift for $\mathrm{C}$ atoms bonded in $\mathrm{sp}^{2}$ rings. The observation of dispersion of the Raman line width implies that there is an inhomogeneous distribution present in the bond angle disorder. Furthermore, the reduction of the $\mathrm{G}$ width with increasing excitation energy implies that there is a narrower distribution of higher gap $\mathrm{sp}^{2}$ states in contradiction with the proposal that there must be a larger distribution of $\mathrm{sp}^{2}$ states 
required for the large Urbach energy. The increase in the Urbach energy with increasing $\pi-\pi^{*}$ separation and small numbers of possible $\mathrm{sp}^{2}$ cluster configurations, coupled with the dispersion in the G line width have lead Fanchini and Tagliaferro to conclude that the Urbach energy in amorphous carbon is not a good measure of disorder [11]. Furthermore, they went onto propose two types of disorder:

(i) a structural disorder associated with clusters of the same size but with different amounts of distortion. This type of inhomogeneous disorder would increase the Urbach energy as well as broaden the G band line width, and

(ii) a topological disorder arising from undistorted clusters but of different sizes. This would also broaden the Urbach energy but have no effect on the G line width.

As a consequence the difference between distorted and undistorted $\mathrm{sp}^{2}$ clusters needs to be explored. The location of the $\mathrm{sp}^{2}$ clusters in the energy gap depends on two factors; whether they consist of even or odd numbers of carbon atoms and whether they are distorted. Undistorted even numbered clusters will give rise to states near the Fermi level $\left(\mathrm{E}_{\mathrm{F}}\right)$ only if they are sufficiently large. This differs from the case of odd numbered clusters which can give rise to gap states even if composed of a small number of atoms. In general, distorted clusters gives rise to states that are closer to $\mathrm{E}_{\mathrm{F}}$ than undistorted clusters. One method that is able to distinguish between the different types of cluster is to measure the density of unpaired electron spins arising, for example, from odd numbered clusters. Such states can be measured by electron paramagnetic resonance (EPR) - also called electron spin resonance (ESR). A high concentration of paramagnetic defects $\left(10^{20} \mathrm{~cm}^{-3}\right)$ is typically found in films deposited under energetic conditions. Such energetic conditions include samples deposited on the driven electrode of a PECVD system. [12] Low defect densities typically $\sim 10^{17} \mathrm{~cm}^{-3}$ tend to be found under less energetic conditions. [13] The term 'defect' is a misnomer since the ESR active species are generally agreed to result from different configurations of $\mathrm{sp}^{2}$ clusters. In this manner, ESR can give a measure of the density of $\mathrm{sp}^{2}$ states at the Fermi level, provided that a majority of these states have a net unpaired electron spin present. It should be noted that ESR spin densities do not themselves measure the $\mathrm{sp}^{2}$ content.

\section{Intracluster Effects in Amorphous Carbon}

Several studies have examined the variation of spin density with deposition conditions, though rarely in terms of relating spin density to localisation of the wavefunction. [2] The variation of the spin density, $\mathrm{N}_{\mathrm{s}}$, and peak-to-peak line width, $\Delta \mathrm{B}_{\mathrm{pp}}$, with negative self-bias for three different series of films are shown Fig. 4. 

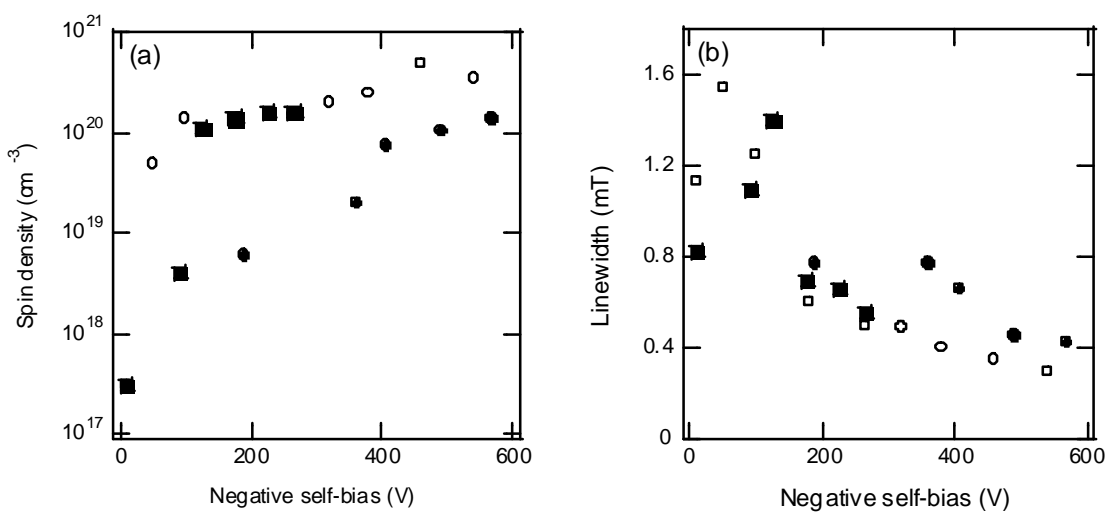

Fig. 4. Variation of (a) spin density and (b) peak-to-peak linewidth with negative self-bias for three series of a-C:H films (see ref [2])

It is evident that the spin density rises from a low value of $\sim 10^{17} \mathrm{~cm}^{-3}$ and tends to saturate at $10^{20} \mathrm{~cm}^{-3}$. The ESR line width initially increases, often as the spin density increases, but at higher biases the line width decreases. In this regime the line shape of the ESR signal is Lorentzian. Furthermore, in these high bias conditions the spin densities are typically $\sim 10^{20} \mathrm{~cm}^{-3}$, corresponding to $\sim 1$ at. $\%$ of the film. As a result, the ESR signal can be considered as originating from a dilute paramagnetic material. Abragam's formulism may be used to calculate the contribution to the peak-to-peak line width, $\Delta \mathrm{B}_{\mathrm{pp}}$ from the dipole-dipole interaction between like spins [14]. Such a broadening mechanism will produce a Lorentzian ESR line shape, which is consistent to the ESR line shapes observed. Assuming a spin concentration $\mathrm{N}_{\mathrm{s}}$, measured in $\mathrm{cm}^{-3}$, the contribution to the dipolar interaction $\Delta \mathrm{B}_{\mathrm{pp}}$, measured in $\mathrm{mT}$, can be given as [12].

$$
\Delta \mathrm{B}_{\mathrm{pp}}=\frac{4 \pi^{2}}{9} \mathrm{~g} \mu_{B} \mathrm{~N}_{\mathrm{s}}
$$

Barklie et al. applied Eq. (1) for a typical $\mathrm{C}$ related $\mathrm{g}$ value of 2.0025 to obtain the expression $\Delta \mathrm{B}_{\mathrm{pp}}=8.12 \times 10^{-21} \mathrm{~N}_{\mathrm{s}}$. The variation of $\Delta \mathrm{B}_{\mathrm{pp}}$ against $\mathrm{N}_{\mathrm{s}}$ directly for the same three data sets reported in Fig. 4, as well the predicted line width (dashed line) based solely on dipolar broadening using Eq. (1) is shown in Fig. 5. It is apparent that significant reductions in $\Delta \mathrm{B}_{\mathrm{pp}}$ are observed at high spin densities indicating an additional mechanism is responsible for reductions in the line width. This additional interaction is due to the motional averaging of the electron wavefunction within the $\mathrm{sp}^{2}$ cluster. As the $\mathrm{sp}^{2}$ cluster increases in size the probability of the electron being associated with a particular atom decreases. This spread out or delocalization of the wavefunction as the cluster size increases is accompanied by 


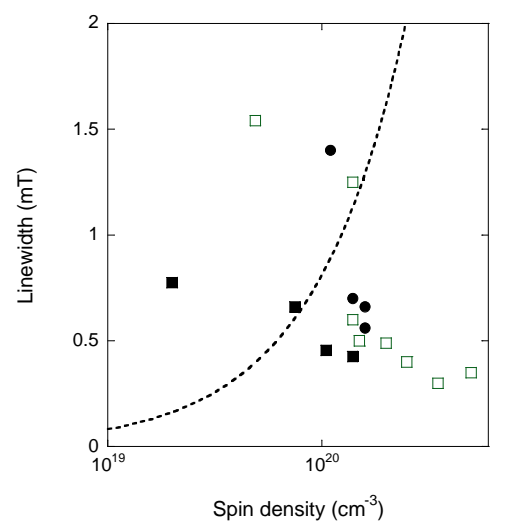

Fig. 5. Variation of peak-to-peak linewidth with spin density for a data in Fig. 4. The dashed line represents the predicted dipolar contribution to the linewidth based on Eq. 1.

a general reduction in the Tauc gap. Further evidence of delocalization can also be found in an examination of the spin resonance relaxation times. The spin-lattice relaxation time, $\mathrm{T}_{1}$, decreases from $3 \times 10^{-5} \mathrm{~s}$ for polymer-like films down to $2 \times 10^{-7} \mathrm{~s}$ for DLC films which implies a greater interaction between the spin system and lattice [12]. At the same time, the spin-spin relaxation times increase reflecting a change in the exchange frequency. It has been reported that in films with a high $\mathrm{sp}^{2}$ content that the exchange frequency is approximately $\sim 10^{10} \mathrm{rad} \mathrm{s}^{-1}$.

In addition to the concentration of the $\mathrm{sp}^{2}$ clusters, the size of the $\mathrm{sp}^{2}$ clusters is therefore an important feature of this description. Indeed there have been attempts to infer the cluster size and shape using visible Raman spectroscopy [15]. As a result considerable interest was generated by the report [16] of an apparent anisotropy in the ESR signal of defects in DLC films measured at high frequency and low temperature. A fully amorphous material with a single spin $\frac{1}{2}$ paramagnetic centre should exhibit a single isotropic line. In reference [16] it was proposed that the ESR signal could be assigned to the sum of an anisotropic powder spectrum with axial $g$ values of $\mathrm{g}_{\|} \sim 2.005$ and $\mathrm{g}_{\perp} \sim 2.0025$ and one symmetric unresolved line with $\mathrm{g}=2.0025$. From previous ESR studies of nanocrystalline graphite it is known that the $\mathrm{g}$ tensor is anisotropic and the $\mathrm{g}$ values depend on the average crystallite size. A value of $g_{\|}$of 2.005 was associated [17] with a crystallite of a size less than 7.5 $\mathrm{nm}$. In this way, it was believed that ESR was providing a way to quantify the size of the nanoclusters. This is an extremely attractive feature since it has been proposed that the ratio of the intensities of the G and D Raman bands (the $\mathrm{D}$ band being located at $1350 \mathrm{~cm}^{-1}$ and associated with the $\mathrm{A}_{1 \mathrm{~g}}$ breathing mode of 6 -fold $\mathrm{sp}^{2}$ rings) can be related to the in-plane correlation 
length of disordered graphite. The reason that no anisotropy was observed at the more conventional $9 \mathrm{GHz}$ room temperature measurement was due to the strong exchange narrowing. Unfortunately, in this analysis the role of demagnetizing fields, which at room temperature and 'low' magnetic fields are negligible, but must be included when operating at $4 \mathrm{~K}$ and especially at higher resonance fields, required at the higher microwave frequencies $[18,19]$. When demagnetizing fields are taken into account, an apparent anisotropy of the resonance can occur since the demagnetizing field results in an effective shift downwards of the effective g value when the applied Zeeman magnetic field is perpendicular to the film and results in an up-shift when the applied field is parallel to the plane.

Apart from changes in deposition conditions, such as self bias or the addition of $\mathrm{N}$, nanoclustering within a-C films can be adjusted via thermal annealing. Siegal et al. annealed non-hydrogenated a-C films produced by pulsed laser ablation and reported the presence of regions $3-5 \mathrm{~nm}$ in size which were $5-10 \%$ more dense that the surrounding regions [20]. The asdeposited films has stresses of 5-7 GPa and a film density of $3.0 \mathrm{~g} \mathrm{~cm}^{-3}$, but that near complete stress relief had occurred and the density had dropped to $2.8 \mathrm{~g} \mathrm{~cm}^{-3}$ with thermal annealing to $600^{\circ} \mathrm{C}$. Over the same annealing temperature range the optical transparency of the film decreased, demonstrating that clustering of the $\mathrm{sp}^{2}$ within the film has occurred. By considering different bonding topologies on the basis of Raman spectroscopy, Siegal et al. concluded that the as-grown films consist of small and isolated sixfold clusters, but that above annealing temperatures of $300^{\circ} \mathrm{C}$ the fraction of five-membered rings begins to increase. The presence of five membered rings was attributed to the newly formed $\pi$ bonded atoms, rather than a reduction in the population of the six membered rings themselves. Despite the reduction in the film's density, nanoindentation measurements showed that the nanocomposite films were $15 \%$ harder than the as-deposited films.

Computational studies showed that within this nanocomposite film, the residual stress consists of an inhomogeneous stress distribution where the three-fold coordinated atoms are under tensile stress but that the four-fold atoms are under a smaller compressive stress [21]. During annealing small regions of high stress relax, leading to the preferential generation of threefold coordinated atoms. The stress relief is as a result of fourfold $\mathrm{sp}^{3} \mathrm{C}$ bonds being replaced by threefold $\mathrm{sp}^{2} \mathrm{C}$ bonds. This change to the shorter $\mathrm{sp}^{2} \mathrm{C}$ bond is irreversible under normal conditions. If the near zero stress is achieved due to high temperature annealing, care must the exercised to prevent an excessive thermal stress being introduced into the film on cooling back to room temperature. The thermal stress can be eliminated by growing the film at above room temperature which introduces some tensile stress. Further evidence of the formation of clustering in ta-C films, was reported in the fluctuation microscopy studies recently undertaken by Chen et al. [22] . Annealing up to $600^{\circ} \mathrm{C}$ resulted in the increase in locally ordered regions but 
the formation of graphitic ordering did not occur until annealing to $1000^{\circ} \mathrm{C}$. Much of the research for stress relief in hard a-C films, especially ta-C films is driven by the development of carbon based microelectromechanical systems.

Evidence for mixed phase components in a-C has also been obtained by examining the proton and ${ }^{13} \mathrm{C}$ relaxation times using nuclear magnetic resonance (NMR). Jäger et al. used cross polarization ${ }^{13} \mathrm{C}$ NMR to measure the effects on the proton relaxation times in different environments of films deposited from acetylene and benzene. [23] A two component ${ }^{1} \mathrm{H}$ spin-lattice relaxation with time constants of 14 and $120 \mathrm{~ms}$ was found and interpreted as being due to the presence of two differently relaxing proton systems. The shorter relaxation time originates from $\mathrm{CH}$ groups in the $\mathrm{sp}^{2}$ and $\mathrm{sp}^{3}$ matrix and the longer $\mathrm{T}_{1}$ time originating from short $\mathrm{CH}_{2}$ polymer units with a sp ${ }^{3}$ configuration. In this description the two environments are separated by regions of non-hydrogenated $\mathrm{sp}^{2} \mathrm{C}$. It is was further found that for films grown by rf glow discharge from benzene at a self-bias voltage of $-200 \mathrm{~V}$, the proton relaxation time of $\sim 10 \mathrm{~ms}$ was virtually independent of temperature. This temperature independence of the shorter $\mathrm{T}_{1}$ component was interpreted in terms of nuclei being relaxed via spin diffusion to paramagnetic centers. It is therefore the degree of electron aggregation or clustering around these paramagnetic centers that determines the proton relaxation. The influence of spin diffusion to paramagnetic centers as an explanation of the temperature independence of the spin-lattice relaxation times implies that an understanding of the factors that affect the spin density and line width are important.

Tamor et al. estimated from NMR measurements the protonated and non-protonated $\mathrm{C}$ concentration with either $\mathrm{sp}^{2}$ or $\mathrm{sp}^{3}$ hybridization [24]. At a self-bias of $-500 \mathrm{~V}$, the $\mathrm{H}$ content was estimated to be 36 at. $\%$ and the fraction of protonated $\mathrm{sp}^{3} \mathrm{C}$ atoms to be 0.16 , non-protonated $\mathrm{sp}^{3} \mathrm{C}$ atoms to be 0.20 , protonated $\mathrm{sp}^{2} \mathrm{C}$ atoms to be 0.25 , and non-protonated $\mathrm{sp}^{2} \mathrm{C}$ atoms to be 0.37 . For a film density of $1.7 \mathrm{~g} \mathrm{~cm}^{-3}$, this result suggests there being $5.1 \times 10^{22} \mathrm{sp}^{2} \mathrm{C}$ atoms $\mathrm{cm}^{-3}$ in the film. If the signal observed from ESR is associated with $\mathrm{sp}^{2} \mathrm{C}$ centers, then for a value of $\mathrm{N}_{\mathrm{s}}$ of $10^{20}$ $\mathrm{cm}^{-3}$, this corresponds to 1 spin per $510 \mathrm{sp}^{2} \mathrm{C}$ atoms or equivalent to 1 spin per 300 atoms of non-protonated $\mathrm{sp}^{2} \mathrm{C}$ atoms. If these spins were randomly distributed throughout the film, co-operative effects, such as exchange, should not occur. Since exchange effects have been attributed to films grown at high bias, the combined use of NMR and ESR confirms and quantifies the clustering of the $\mathrm{sp}^{2}$ phase has taken place.

\section{Intercluster Interactions in Amorphous Carbon}

The increase in intracluster delocalization also manifests itself in changes that can be associated with intercluster interactions. Various transport studies of a-C films reveal a commonality in behaviour with high resistivity associated with samples with large Tauc gap. The resistivity decreases as the Tauc gap 
decreases and the conductivity associated with various mechanisms such as variable range hopping in the more conductive DLC films [1]. In the case of resistive polymer-like amorphous carbon the conductivity has been attributed to a space charge induced current mechanism [1]. Improvements in the conductivity have also been reported with nitrogen incorporation and attributed to a weak 'doping' effect [25] or to a reduction of the band gap through graphitization [26]. In terms of post deposition processing, transport studies of annealed films also reveal an improvement in the conductivity. However, this is almost always accompanied by significant modification to the structure of the film at a microscopic level. In this respect furnace annealing results in an improvement in electrical properties but accompanied by global changes to the film. Ion implantation as a method of injection of thermal energy is therefore an alternative process to modify the film properties in a highly controlled manner with precise depth control by the correct choice of ion species, ion energy and dose. Using high dose $\mathrm{B}^{+}$implantation into polymerlike films, it is possible to reduce the low field resistivity from $2 \times 10^{14} \Omega \mathrm{cm}$ (unimplanted) to $6 \times 10^{12} \Omega \mathrm{cm}\left(2 \times 10^{14} \mathrm{~B}^{+} \mathrm{cm}^{-2}\right)$. Over the same range the Tauc gap remained constant at about $2.6 \mathrm{eV}$, indicating that no significant increase in the mean $\mathrm{sp}^{2}$ cluster size has occurred. For doses greater than $2 \times 10^{14} \mathrm{~cm}^{-2}$, a fall in the optical gap finally ends with a total collapse to $0.2 \mathrm{eV}$ at a high dose $\left(2 \times 10^{16} \mathrm{~B}^{+} \mathrm{cm}^{-2}\right)$ and the resistivity concomitantly decreases to $5 \times 10^{6} \Omega \mathrm{cm}$. The improvement in the electrical characterization has been attributed to improvements in the cluster-cluster interaction via a hopping related mechanism as shown in Fig. 6.

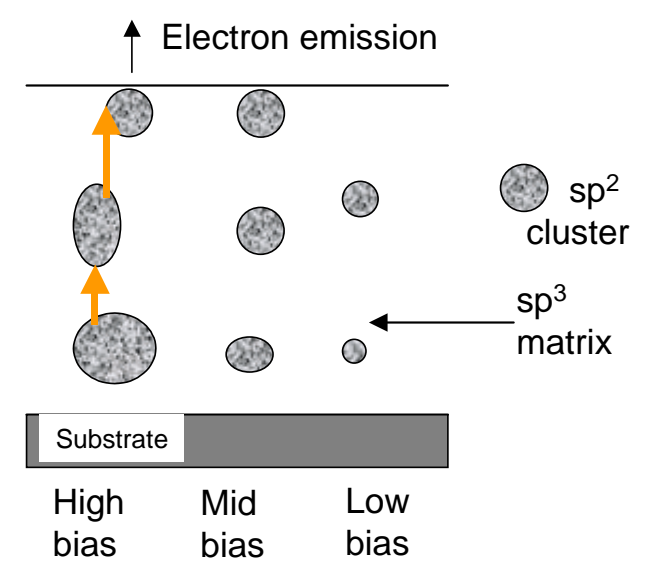

Fig. 6. Schematic of how the different bias growth conditions can give rise to a change in the size and concentration of $\mathrm{sp}^{2}$ clusters. 
During low dose implantation, localized heating results in localized $\mathrm{sp}^{2}$ clusters, which remain small (as evidenced by the absence of a reduction in the Tauc gap). Before the formation of damage cascades, the concentration of the induced sites will be proportional to the ion dose. This nanoclustering of the $\mathrm{sp}^{2}$ sites, which maintains the wide gap, increases the conductivity by decreasing the hopping distance. At higher doses, nanostructuring of the bulk of the film occurs and the band gap decreases. As a result, ion implantation demonstrates that it is possible to improve the conductivity without inducing extensive graphitizing of the film.

Further evidence for the idea of dielectric inhomogeneity of conductive $\mathrm{sp}^{2}$ clusters embedded in a more insulating matrix can be found in the high resolution scanning tunneling microscope for a DLC film, where the STM results show that there are nanometer sized regions of higher conductivity surrounded by other regions of lower conductivity [2]. Similar localized regions of conductivity have also been recently reported in an STM study of sulphur containing ultrananocrytalline diamond films (UNCD). [27] UNCD films consist of nm sized grains surrounded by graphitic grain boundaries. The surface density of states was determined from the normalized differential conductivity which for $\mathrm{S}$ rich films showed an oscillatory behaviour. In UNCD films, it was reported that the $\mathrm{S}$ acts both to dope the films but also to introduce localized defects. In both cases increasing the concentration and size of the clusters results in improved localized electrical conductivity. This is an important result since conventional 'large area' contacts used in currentvoltage characteristics would mask the localized nature of the conductivity.

\section{Field Emission from Amorphous Carbon}

The implication of this 'dielectric inhomogeneity' can also be extended to examine the field induced electron emission from a-C:H films. Application of an applied electric field can result in electron emission from the surface. In the case of PAC, films it has been reported [28] that the threshold electric field for emission exhibited a dependence with the film thickness with a minimum threshold observed for a thickness of $65 \mathrm{~nm}$. This behavior was attributed to the effects of internal high electric field effects within the film and the absence of any significant screening of the applied electric field. Such an explanation is possible when one considers that the electric field from the anode will terminate on the 'more conductive' $\mathrm{sp}^{2}$ clusters. At a low density $\left(\sim 10^{17}\right.$ $\mathrm{cm}^{-3}$ ) screening of the bulk of the film will not occur. In such a situation the controlling step in the electron emission process is at the film/substrate. A different situation will result for films grown at higher biases when the defect density increases. For two films deposited at $-90 \mathrm{~V}$ and $-265 \mathrm{~V}$ self bias, it was observed [29] that there is little dependence on the film thickness and the lowest threshold field was reported for the film grown at the highest bias, consistent with the model presented in Fig. 6 . Here, the film with the 
largest bias has the greatest inter-cluster interactions since the clusters are larger and the cluster-cluster separation will be the lowest. This gives rise to an emission mechanism which is dominated by the front surface properties of the film. Furthermore, the local electric field in the neighbourhood of the clusters is enhanced, resulting in lower than predicted threshold fields for emission.

Finally, it has been reported in several studies that the onset of stable field emission, often in more resistive samples, occurs only after several voltage cycles [30,31]. This conditioning behaviour often results in lower threshold fields. It has been shown that it is possible to condition a PAC film by current stressing, by intentionally passing a current of suitable magnitude through the film [31]. Mercer et al. performed a similar experiment using a scanning tunneling microscope tip to generate a highly spatially localized electric field and effectively current stress ta-C films [32]. They observed that after ramping the tip-sample bias and current, nanostructures of about $100 \mathrm{~nm}$ in extent form. By using high resolution spatially-resolved electron energy loss spectroscopy (EELS), they showed that the predominant bonding configuration changes from predominately fourfold coordinated $\mathrm{C}$ to threefold coordination. Changes in the $\mathrm{sp}^{2}$ bonding have also been reported for heavy ion irradiation of S doped UNCD films. [33] High energy ( GeV/amu) Fe and Si ions were use and it was reported that there was a reduction in the threshold field after implantation. for films irradiated and this improvement in threshold field was accompanied by changes in the $\mathrm{sp}^{2}$ phase as inferred from by Raman spectroscopy. The net effect of the conditioning, current stressing or heavy ion treatment, is to generate conductive $\mathrm{sp}^{2}$ rich areas or filaments through localized Joule heating. These conductive regions can then be viewed as either isolated $\mathrm{sp}^{2}$ clusters or a continuous $\mathrm{sp}^{2}$ network which can lead to high field enhancement factors, which result in reduced threshold fields.

Further evidence of the importance of the $\mathrm{sp}^{2}$ phase in field emission comes from a comparative study of the threshold field with the ratio of the $\mathrm{I}_{\mathrm{D}} / \mathrm{I}_{\mathrm{G}}$ bands found in Raman spectroscopy. For a range of different types of a-C films (including ta-C, ta-C:N, ta-C:H and DLC films [16] and $\mathrm{S}$ doped UNCD films [34]) it was observed that the threshold field is correlated to the in-plane correlation length suggesting $\mathrm{sp}^{2}$ cluster sizes of around 1-2 $\mathrm{nm}$. The in-plane correlation length is inferred from the ratio of the intensities of $D$ and G Raman bands.

\section{Conclusions}

In conclusion, the disorder inherent in amorphous carbon films plays a major role in determining the electronic properties. An understanding of the role of disorder is important. PAC films have small clusters with a small distribution of cluster sizes and dominated by topological disorder in which the Urbach energy is not a good measure of disorder and topological disorder dominates. 
In DLC films a reduction in the Tauc gap and blue shift of the $G$ band implies a higher concentration of larger clusters is present. Furthermore the increase in spin density $\left(10^{20} \mathrm{~cm}^{-3}\right)$ coupled with the increase in the $\mathrm{G}$ band width implies more distorted clusters are present. At the higher spin density the ESR line width is no longer determined by the dipole interaction, with intracluster exchange interactions determining the line width. In this regime, both topological and structural disorder is present and can effect the stress in the film. The disorder is dominated by structural disorder with little topological disorder thereby reducing the line width of $\mathrm{G}$ band. The clustering can be adjusted by thermal annealing or ion implantation. In addition the $\mathrm{sp}^{2}$ phase plays an important role in the field emission from these films.

\section{Acknowledgements}

The authors acknowledge funding from the EPSRC and JDC acknowledges the funding of an Advanced Research Fellowship from the EPSRC. 


\section{References}

1. S. R. P. Silva, J. D. Carey, R. U. A. Khan, E. G. Gernster, and J. V. Anguita, in: Handbook of Thin Film Materials, H. S. Nalwa, ed. (Academic Press, New York, 2002), and reference therein.

2. J. D. Carey and S. R. P. Silva, Phys. Rev. B. 70, 235417 (2004).

3. D. Dasgupta, F. Demichelis, C. F. Pirri, and A. Tagliaferro, Phys. Rev. B. 43, 2131 (1991).

4. G. Fanchini, S. C. Ray, and A. Tagliaferro, Diam. Relat. Mater. 12, 891 (2003).

5. A. C. Ferrari and J. Robertson, Phys. Rev. B. 61, 14095 (2000).

6. J. Ristein, J. Schafer, and L. Ley, Diam. Relat. Mater. 4, 509 (1995).

7. A. Zeinert, H.-J. von Bardeleben, and R. Bouzerar, Diam. Relat. Mater. 9, 728 (2002).

8. F. Giorgis, F. Giuliani, C. F. Pirri, and A. Tagliaferro, Appl. Phys. Lett. 72, 2520 (1998).

9. M. A. Tamor and W. C. Vassell, J. Appl. Phys. 76, 3823 (1994).

10. J. Robertson, Mater. Sci. Eng. R 37, 129 (2002).

11. G. Fanchini and A. Taliaferro, Appl. Phys. Lett. 85, 730 (2004).

12. R. C. Barklie, M. Collins, and S. R. P. Silva, Phys. Rev. B. 61, 3546 (2000).

13. M. Collins, R. C. Barklie, J. V. Anguita, J. D. Carey, and S. R. P. Silva, Diam. Relat. Mater. 9, 781 (2002).

14. A. Abragam, in: Principles of Nuclear Magnetism (Clarendon, Oxford, 1996).

15. A. Ilie, A. C. Ferrari, T. Yagi, and J. Robertson, Appl. Phys. Lett. 76, 2627 (2000).

16. H. J. von Bardeleben, J. L. Cantin, A. Zeinert, B. Racine, K. Zellama, and P. Hai, Appl. Phys. Lett. 78, 2843 (2001).

17. G. Wagoner, Phys. Rev. 118, 647 (1960).

18. B. Druz, I. Zaritskiy, Y. Evtuikhov, A. Konchits, M. Y. Valakh, S. P. Kolesnik, B. D. Shanina, and V. Visotskij, Mat. Res. Soc. Symp. Proc. 593, 249 (2000).

19. B. J. Jones, R. C. Barklie, G. Smith, H. E. Mkami, J. D. Carey, and S. R. P. Silva, Diam. Relat. Mater. 12, 116 (2003).

20. M. P. Siegal, D. R. Tallant, P. N. Provencio, D. L. Overmyer, R. L. Simposn, and L. J. Martinez-Miranda, Appl. Phys. Lett. 76, 3052 (2000).

21. P. C. Kelires, Phys. Rev. B. 62, 15686 (2000).

22. X. Chen, J. P. Sullivan, T. A. Friedmann, and J. M. Gibson, Appl. Phys. Lett. 84, 2823 (2004).

23. C. Jäger, J. Gottwald, H. W. Spiess, and R. J. Newport, Phys. Rev. B. 50, 849 (1994).

24. M. A. Tamor, W. C. Vassell, and K. Carduner, Appl. Phys. Lett. 58, 592 (1991).

25. G. A. J. Amaratunga and S. R. P. Silva, Appl. Phys. Lett. 68, 2529 (1996).

26. R. U. A. Khan, J. D. Carey, S. R. P. Silva, B. J. Jones, and R. C. Barklie, Phys. Rev. B. 63, 121201 (2001).

27. S. Gupta, B. R. Weiner, and G. Morell, J. Appl. Phys. 97, 094307 (2005).

28. R. D. Forrest, A. P. Burden, S. R. P. Silva, L. K. Cheah, and X. Shi, Appl. Phys. Lett. 73, 3784 (1998).

29. J. D. Carey, R. D. Forrest, and S. R. P. Silva, Appl. Phys. Lett. 78, 2339 (2001).

30. A. A. Talin, T. E. Felter, T. A. Friedmann, J. P. Sullivan, and M. P. Siegal, J. Vac. Sci. Technol A. 14, 1719 (1996).

31. J. D. Carey and S. R. P. Silva, Appl. Phys. Lett. 78, 347 (2001). 
32. T. W. Mercer, N. J. DiNardo, J. B. Rothman, M. P. Siegal, T. A. Friedmann, and L. J. M. Miranda, Appl. Phys. Lett. 72, 2244 (1998).

33. A. Gonzalez-Berrios, D. Huang, N. M. Medina-Emmanuelli, K. E. Kristian, O. O. Ortiz, J. A. Gonzalez, J. D. Jesus, I. M. Vargas, B. R. Weiner, and G. Morell, Diam. Relat. Mater. 13, 221 (2004).

34. S. Gupta, B. R. Weiner, and G. Morell, Appl. Phys. Lett. 80, 1471 (2002). 